\title{
Corela
}

Cognition, représentation, langage

6-1 | 2008

Vol. $6, n^{\circ} 1$

\section{Locutions en SI et marqueurs dans la protase : Compositionnalité et indices co-textuels}

\author{
Jeanne Aptekman
}

\section{OpenEdition}

\section{Journals}

Édition électronique

URL : http://journals.openedition.org/corela/224

DOI : $10.4000 /$ corela.224

ISSN : $1638-573 \mathrm{X}$

Éditeur

Cercle linguistique du Centre et de l'Ouest - CerLICO

Référence électronique

Jeanne Aptekman, «Locutions en SI et marqueurs dans la protase : Compositionnalité et indices cotextuels », Corela [En ligne], 6-1 | 2008, mis en ligne le 24 juin 2008, consulté le 19 avril 2019. URL : http://journals.openedition.org/corela/224 ; DOI : 10.4000/corela.224

Ce document a été généré automatiquement le 19 avril 2019

\section{(c) (i) (2)(2)}

Corela - cognition, représentation, langage est mis à disposition selon les termes de la licence Creative Commons Attribution - Pas d'Utilisation Commerciale - Partage dans les Mêmes Conditions 4.0 International. 


\title{
Locutions en SI et marqueurs dans la protase : Compositionnalité et indices co-textuels
}

\author{
Jeanne Aptekman
}

\section{Introduction}

1 Il s'agit pour nous ici de rendre compte d'une distinction entre deux types de locutions en SI : celles qui finalement forment ce que l'on pourrait appeler, en suivant M. Piot ${ }^{1}$, une conjonction et celles qui, sans former une telle conjonction, ne font que renforcer une valeur de SI qui peut être construite en l'absence d'un tel marqueur.

2 M. Piot $^{2}$ propose une analyse de la locution même si selon laquelle même doit être analysé comme ce qu'elle appelle un modificateur du sens de SI (qui garderait à ce titre une interprétation habituelle, augmentée en quelque sorte des effets de sens de même), au même titre que seulement ou précisément - elle en conclut, selon le titre de son article, que la conjonction même SI n'existe pas. Nous ne partageons pas cette partie de l'analyse : nous pensons que seulement (placé avant le SI) permet d'obtenir un usage nouveau de SI, tandis que même ne fait que renforcer un usage déjà existant de SI. Cependant c'est la deuxième partie de son analyse qui nous intéresse ici.

En effet, M. Piot souhaite ainsi s'opposer à l'idée répandue ${ }^{3}$ selon laquelle même SI correspondrait à une conjonction de subordination, classée avec bien que, ou quoique. Pour M. Piot, au contraire, même modifie le sens de SI, et c'est le mot même qui est porteur de la concession, et non la conjonction même SI. Nous souscrivons à cette analyse dans le sens où nous distinguons clairement même SI de conjonction telles que bien que ou quoique.

Cependant, nous voudrions développer l'idée selon laquelle, si même garde ici sons sens, il en va de même pour SI, dont le sens nous semble préservé dans les constructions en même SI. Ainsi, nous voudrions montrer comment il est possible d'obtenir des usages concessifs avec SI, en l'absence de tout autre marqueur concessif, et que dans la locution même SI, même ne fait que forcer l'une des interprétations possibles de SI. 
Examinons en effet l'exemple suivant :

(1) SI Jake et Chuckie sont les personnages centraux du roman, ce serait faire injure à Mac Liam Wilson d'oublier la palette d'individus tous plus extravagants les uns que les autres. (Critique du roman Eurêka Street de Mc Liam Wilson sur un site Internet consacré à l'auteur)

Dans cet exemple, la concession n'est portée par aucun marqueur concessif, et pourtant, le sens concessif apparaît clairement.

7 Il ne s'agit pas pour autant de dire que c'est le SI qui pousse à une telle interprétation concessive, mais plutôt qu'il la rend possible dans certains types de rapports entre la protase et l'apodose que nous développerons.

8 Ainsi, plus que de modifier le sens de SI, même contribue selon nous à forcer l'une des interprétations possibles de SI, dans les cas où les éléments qui poussent à une telle interprétation concessive de SI ne sont pas assez saillants dans le contexte.

Nous voudrions donc essayer de distinguer les cas où un usage avéré de SI est renforcé par la présence récurrente d'un marqueur (qui pointerait ainsi vers l'une des interprétations de SI) des cas où la présence d'un tel marqueur permet la formation d'une conjonction ayant un sens nouveau, obtenu par compositionnalité, et que le SI n'aurait pas suffi à construire.

10 Nous donnerons ici un certain nombre d'exemples pour marquer cette différence. Il est certain qu'une telle étude mériterait d'être ensuite développée, et nous n'entrerons pas ici dans l'analyse détaillée des marqueurs associés à SI. Notre choix méthodologique consiste simplement à essayer de déterminer si le marqueur associé à SI permet d'obtenir une valeur de SI qui ne saurait être obtenue en son absence ou s'il ne fait que renforcer une valeur avérée de SI.

11 Nous nous intéressons ici tout particulièrement au SI, et nous nous appuierons sur notre analyse du sens de SI, qui propose une classification des valeurs possibles de SI. Nous ne prétendons pas fournir une analyse détaillée des autres marqueurs auxquels il sera fait allusion, cependant, cette approche nous semble également permettre de fournir quelques pistes sur ceux-ci.

\section{Cadre théorique général : classification des valeurs de SI}

12 Avant d'étudier le rôle d'éventuels marqueurs associés à SI, nous voudrions introduire notre classification des usages de SI et les arguments qui ont présidé à son développement. Il ne s'agit pas ici de rentrer dans les détails de cette classification, mais plutôt d'en présenter les lignes directrices et les enjeux.

\section{Méthodologie générale}

\subsubsection{Deux idées directrices : continuité du sens et noyau de sens instructionnel}

13 Nous voudrions dans notre analyse tenir compte de deux hypothèses, qui sont liées. Le premier point qui nous semble important, dans la lignée des études sur la polysémie ${ }^{4}$, est l'idée d'une continuité du sens de SI. Une telle idée semble justifiée par le fait que dans 
certains cas, plusieurs interprétations semblent possibles sans pour autant être contradictoires. exemple peut être compris comme mettant en jeu une valeur de simple comparaison entre deux moments différents, ou être interprété, du fait du caractère prospectif des constructions, comme formulant une sorte d'enchaînement - déjoué entre protase et apodose. Nous reviendrons plus longuement sur ces caractéristiques dans les paragraphes suivants, mais ce qui nous intéresse ici c'est cette possibilité de cohabitation de plusieurs sens, qui manifeste une sorte de continuité des valeurs qui nous aurait sans doute échappée si nous nous en étions tenus à des exemples construits, ou si nous avions essayé de n'observer que des exemples « simples ». Il n'est pas forcément ici question d'ambiguiité, mais plutôt d'un recoupement de valeurs, qui donne une grande richesse au sens de l'énoncé. C'est ce type de phénomènes que nous voudrions rendre compte en parlant de continuité du sens. les grammaires cognitives, selon lesquelles le SI serait un constructeur d'espace. En ce sens, nous voudrions l'analyser d'un point de vue instructionnel, en nous posant deux types de questions :

- Quels types d'espaces peuvent être construits par SI?

- Quels éléments influent sur la construction de ces espaces?

Nous proposons ainsi de comprendre SI comme une forme sous-déterminée dont la spécification dépend de facteurs cotextuels divers mais globalement prédictibles. Il ne s'agit donc pas pour nous de gommer ou de sous-estimer ces effets contextuels, mais de comprendre comment ils sont possibles.

\subsubsection{Etude sur corpus}

21 Partant de ce cadre théorique général, nous avons choisi de travailler sur un corpus de textes, afin d'aller examiner dans la langue les occurrences du SI pour essayer de caractériser ses usages possibles. Ce choix correspond à un parti pris : il s'agit non pas de travailler sur des exemples construits, mais d'examiner des exemples réels, extraits de textes, essentiellement littéraires, mais pas uniquement, puisque nous nous sommes aussi intéressé à des essais, des critiques, des rapports, etc. ${ }^{6}$. 

L'analyse d'un corpus oral semblerait indispensable si l'on s'attachait à établir des statistiques sur la fréquence d'usage de telle ou telle valeur de SI, ce qui n'était pas notre objet ici. On pourrait alors s'attendre à ce que certains usages, qui nous semblent correspondre à un niveau de langue plus élevé, comme les usages contrastifs, y soient peu représentés. Cependant, nous ne pensons pas qu'elle permettrait nécessairement de dégager de nouvelles valeurs.

Nous avons constitué notre corpus à partir de plusieurs sources. D'une part, nous avons utilisé le logiciel Frantext ${ }^{7}$ qui nous a permis de répertorier des phrases en SI dans le corpus mis en ligne par le laboratoire ATILF, en nous concentrant sur quelques œuvres, qu'elles soient littéraires ou non.

Nous avons complété ces références à l'aide de nos propres lectures, de journaux ou de romans, et nous n'avons pas hésité à ajouter d'autres exemples qui nous semblaient intéressants à cette première base de données quand nous en avons rencontrés. Enfin, nous avons pris en compte des extraits de blogs ou de sites Web, qui constituent ainsi des usages sans doute plus relâchés, et certainement plus actuels.

\section{Remarques sur le corpus.}

Tout d'abord, nous avons fait le choix méthodologique de garder pour chaque phrase étudiée uniquement le contexte fourni par cette seule phrase. Cela aboutit évidemment à des ambiguïtés, qui pourraient éventuellement être résolues si le contexte entier était disponible. Mais pour prendre en compte un tel contexte, d'autres problèmes méthodologiques surgissent : en effet il s'agit d'être capable de déterminer les limites du contexte qui nous semble pertinent. Or de telles limites ne sont pas évidentes à déterminer. Le contexte pertinent pour résoudre l'ambiguïté peut apparaître dans les phrases qui précèdent ou suivent immédiatement celle qui nous occupe, mais il peut également être fourni par des informations développées dans le texte tout entier, par exemple pour les romans, dans lesquels il peut consister dans le caractère du personnage qui intervient, ou dont il s'agit, ou dans les relations entre plusieurs personnages. Ainsi, un énoncé comme :

(3) SI elle n'a pas posé vivante devant le peintre qui l'a retracée, elle a posé dans une mémoire ravivée par le souvenir de l'affreuse catastrophe qui fut sa fin.

peut selon nous être compris de deux façons. Il peut exprimer soit une alternative, rendue par la reformulation (3b), qui n'est possible que si l'on ne sait pas ce qu'il en est, soit exprimer une valeur concessive, rendue dans (3c) par une paraphrase du SI en bien que :

(3b) Soit elle a posé vivante devant le peintre qui l'a retracée, soit elle a posé dans une mémoire ravivée par le souvenir de l'affreuse catastrophe qui fut sa fin.

(3c) Bien qu'elle n'ait pas posé vivante devant le peintre qui l'a retracée, elle a posé dans une mémoire ravivée par le souvenir de l'affreuse catastrophe qui fut sa fin.

Pour pouvoir trancher entre ces deux hypothèses, il faut être capable de déterminer ce que l'on sait au moment où cette phrase est énoncée. Or, pour savoir si une telle information a été donnée, il ne suffit pas forcément de se référer aux phrases qui précèdent ou suivent immédiatement celle présentée : il faudrait être en possession de toutes les informations proposées au lecteur depuis le début du roman. C'est cette raison, 
qui concerne les limites de la détermination d'un contexte pertinent pour la désambiguïsation, qui nous a poussés à ne sélectionner que la phrase dans laquelle le SI apparaissait. d'éléments viennent en quelque sorte influencer l'interprétation du SI. Ils peuvent apparaître comme des éléments parasites, qui n'auraient pas leur place dans des exemples construits. Les phrases sont souvent assez longues, et parfois compliquées. Cependant, ce sont justement de telles phrases que nous comprenons - et construisons. Dans ces phrases, hormis dans les cas d'ambiguïté que nous venons d'évoquer et qui sont dus justement à un manque de contexte, nous sommes capables de comprendre le sens de SI. C'est donc au sein de tels exemples qu'il nous semble légitime d'aller chercher les éléments qui guident notre interprétation, qui serait rendu sans doute plus difficile en l'absence de tels marqueurs. Ainsi, nous ne disons pas que c'est le SI lui-même qui charrie cette diversité de sens. il semble au contraire mettre en place une information - ou une instruction - commune dans tous ces exemples, qui se voit modifiée en fonction d'autres éléments de la phrases, qui peuvent être lexicaux, ou alors consister en des constructions aspectuo-temporelles ou syntaxiques.

L'utilisation d'un corpus constitué de phrases réelles, y compris quand elles apparaissent complexes et mettant en jeu différents facteurs, et prises en elles-mêmes, nous semble donc justifiée, pour les raisons que nous venons d'évoquer: elles permettent de déterminer les éléments qui jouent en faveur d'une interprétation donnée pour SI dans la phrase. Les cas d'ambiguïtés correspondent à des cas où ces éléments ne sont pas concluants, et où un contexte plus large serait requis : ils nous indiquent également que les éléments qui apparaissent dans la phrase ne permettent pas à eux seuls d'isoler une valeur pour SI, mais fonctionnent plutôt comme un ensemble d'indices. Enfin, cette approche permet de mettre en lumière la grande continuité à l'œuvre entre les différentes valeurs du SI.

31 Cela ne nous a cependant pas empêchés d'avoir recours à des exemples construits pour expliciter notre propos quand cela s'est avéré nécessaire.

\subsubsection{Analyse du corpus : deux critères distinctifs}

Nous voudrions désormais présenter la façon dont nous avons abordé ce corpus pour l'analyser.

Nous avons séparés trois catégories de critères. La première concerne les formes de surface et regroupe de éléments qui nous semblent automatiquement calculables. La seconde concerne les traits sémantiques, qui devraient - normalement - pouvoir être dégagés à partir des formes de surface. Enfin, nous avons dégagé des critères plus directement liés au sens global, comme le type de paraphrases ou de reformulations possibles, ou la prise en charge de la protase par le locuteur ${ }^{8}$, sur laquelle nous allons revenir.

Nous voudrions nous arrêter sur deux de ces critères qui nous sont apparus comme centraux. L'un appartient à la catégorie des traits sémantiques, et concerne le type d'articulation entre protase et apodose, et l'autre appartient à la catégorie du sens et concerne la prise en charge de la protase par le locuteur. 


\section{La prise en charge de la protase par le locuteur}

en charge ne dépend pas du SI lui-même, qui ne marque aucune prise en charge particulière, mais nous considérons qu'il n'y a pas d'incompatibilité totale entre les constructions en SI et une valeur positive de prise en charge de la protase. Nous reconnaissons cependant que le marquage linguistique d'une telle prise en charge positive de la protase est beaucoup moins convaincant que le marquage d'une valeur négative de prise en charge de la protase, à l'aide de formes verbales distanciées. que la protase est prise en charge de manière complètement positive, de la même manière que la prise en charge de la protase peut apparaître comme totalement négative dans une phrase comme :

(8) Il ne s'en servira sans doute pas plus qu'hier, mais on ne sait jamais : SI, hier soir, il l'avait eu sur lui quand il est remonté de la salle à manger, il s'en serait bel et bien servi. (Alain Robbe-Grillet, Les Gommes, 1952) 

articulation entre protase et apodose, qui nous est finalement apparu comme le point central pour déterminer la valeur de SI à l'œuvre dans une phrase. Dans les exemples que nous avons cités, on voit déjà que le lien entre protase et apodose peut prendre des formes variées. C'est la diversité de ces formes que nous entendons sous le terme générique d'articulation. Si dans certains cas, on semble avoir affaire à un enchaînement entre la protase et l'apodose, ce n'est pas toujours le cas. Ainsi, dans l'exemple (14), on ne voit pas bien ce qui permettrait de penser un enchainement entre protase et apodose. Un lien est bien sûr créé par le SI, mais il ne saurait être compris comme un lien d'enchaînement. Ainsi, cette notion d'articulation ${ }^{11}$ est volontairement très floue : elle cherche à prendre en compte une idée assez large selon laquelle la protase (ce qui est énoncé dans la protase) rend possible l'apodose (ce qui y est énoncé, ou simplement le fait qu'elle puisse être énoncée). Comme nous allons le voir, cette articulation peut prendre plusieurs formes : ce qui est énoncé dans la protase peut déclencher ce qui est énoncé dans l'apodose (exemple (15)); ce qui est énoncé dans la protase peut rendre malgré tout possible ce qui est énoncé dans l'apodose (exemple 3 dans une interprétation concessive); ou encore l'énonciation de la protase peut rendre possible (pertinente ou acceptable) celle de l'apodose (exemple (14)).

(10) S'il vient, je serai ravie !

(11) SI tu as besoin d'aide, je m'appelle Jeanne.

(3) Si elle n'a pas posé vivante devant le peintre qui l'a retracée, elle a posé dans une mémoire ravivée par le souvenir de l'affreuse catastrophe qui fut sa fin.

C'est la présence d'un tel lien, et sa caractérisation, dont nous avons voulu rendre compte selon les valeurs que nous rencontrions. 

déjoué dans l'énoncé :

(14) SI, sur le plan industriel, elle est remarquable par un effort sérieux d'équipement et de modernisation, en revanche, dans le domaine économico social, elle présente une structure assez archaïque. (L'industrie des conserves en France, 1950)

- Les cas où la protase rend l'énonciation de l'apodose pertinente ou acceptable :

(15) Le reste, SI l'on peut parler de reste quand il est question d'une jeune fille, baignait dans le flot gris de la robe, sauf les galets des coudes, des épaules et des genoux (Hervé Bazin, La Mort du petit cheval, 1950)

53 - Le cas où l'on ne fait pas référence à un rapport d'enchaînement, mais à une mise en relation de deux propositions pour les comparer :

(16) SI, sur le plan industriel, elle est remarquable par un effort sérieux d'équipement et de modernisation, en revanche, dans le domaine économico social, elle présente une structure assez archaïque. (L'industrie des conserves en France, 1950)

54 Nous allons maintenant nous attacher à examiner ces différents types de rapports entre protase et apodose, en fonction desquels nous allons proposer une classification des usages de SI.

\subsection{Présentation de la classification}

La classification que nous proposons s'appuie sur cette idée de rapport de la protase et de l'apodose et reprend les cinq catégories que nous avons distinguées. Cela met en jeu une vision de SI selon la quelle la protase introduite par SI fournit un cadre transitoire pour l'interprétation de l'apodose, qui peut être de plusieurs ordres.

\subsubsection{Cadre énonciatif (valeurs énonciatives)}

Dans le cas des valeurs énonciatives pour les phrases en SI, le rapport entre la protase et l'apodose consiste dans le fait que l'énonciation de la protase peut rendre possible celle de l'apodose. Cette possibilité est une possibilité d'énonciation : la protase peut soit fournir des conditions d'acceptabilité de l'apodose, et dès lors accepter la protase est une condition pour reconnaître l'apodose comme vraie (valeurs métalinguistiques et métadiscursives), soit rendre pertinente l'énonciation de l'apodose (valeurs d'actes de 
discours). En ce sens, elle peut être comprise comme un cadre d'énonciation pour l'apodose, qui fixe en effet les conditions d'acceptabilité ou de pertinence de ce qui va être énoncé dans l'apodose, à travers une sorte de convention proposée par le locuteur (SI l'on peut dire, etc.).

On distingue des réserves métalinguistiques qui portent sur un terme de l'apodose (exemples 17 à 19), les cas où la protase formule une réserve sur l'apodose toute entière (exemples 20 à 22), des emplois métalinguistiques et métamétaphoriques, selon l'appellation de Dancygier et Sweetser (Dancygier et Sweetser 2005) (exemples 23 et 24), et des conditionnels d'acte de discours (exemple 25).

\section{Réserve métalinguistique portant sur un terme de l'apodose, ou sur l'apodose toute entière}

Dans le cas des réserves métalinguistiques qui portent sur un terme de l'apodose, le cadre d'énonciation mis en place par la protase consiste en une sorte de convention sur le caractère hasardeux, ou osé, d'un terme employé, il fonctionne comme une mise entre guillemets : en ceci, on est vraiment dans le domaine du métalinguistique.

(17) Mais l'immanence en mouvement est, SI l'on peut dire, athéisme provisoire.

(A. Camus, L'Homme révolté)

(18) Carmen avait de la vie sa philosophie qui n'était pas amère, elle acceptait son sort, SI l'on peut dire, d'un pied léger et elle se défendait farouchement de tout attachement qui aurait nui à son humeur. (Marguerite Duras, Un barrage contre le Pacifique)

(19) Le reste, SI l'on peut parler de reste quand il est question d'une jeune fille, baignait dans le flot gris de la robe, sauf les galets des coudes, des épaules et des genoux. (Hervé Bazin, La Mort du petit cheval)

\section{Réserve métalinguistique portant sur l'apodose toute entière}

Dans les cas où la protase formule une réserve sur l'apodose toute entière, il s'agit de restreindre la portée de l'apodose, qui, si elle n'était pas acceptée, rendrait fausse l'apodose. Il s'agit donc en quelque sorte de fournir un cadre exceptif pour l'énonciation de l'apodose, qui marque les limites de sa portée.

(20) Il manquait de conscience et, SI l'on excepte son amitié pour Robert, il n'avait pas de fidélité. (Georges Bataille, l'abbé C).

(21) Ils le sont, effectivement, SI l'on fait abstraction du commerce des conserves de poissons : en effet, le commerce des conserves de légumes, fruits et viandes, est largement bénéficiaire. (L'Industrie des conserves en France).

(22) SI j'en juge par ton intérieur, tu ne dois pas gagner lourd. (Hervé Bazin, La Mort du petit cheval)

60 Certes, ici, le caractère exceptif est également porté par d'autres termes, mais la protase n'en fournit pas moins un cadre qui limite la validité de ce qui est énoncé dans la protase. Ce caractère exceptif n'est donc évidemment pas porté par le SI lui-même, mais cela montre que le SI peut introduire un cadre d'interprétation pour la protase, comme dans les exemples métalinguistiques que nous allons désormais présenter.

\section{Conditionnels métalinguistiques et métamétaphoriques à la Dancygier et Sweetser}

61 Dancygier et Sweetser isolent des emplois qu'elles qualifient de métalinguistiques (notre exemple 23), qu'elles séparent des usages métamétaphoriques (24). 
62 Selon nous, ces deux types de phrases doivent se comprendre, au sein des usages énonciatifs comme posant l'un et autre un cadre qui consiste en une convention de traduction, ou plus exactement sur la mise en place d'un mapping, au sein duquel doit se comprendre l'apodose.

(23) SI nous parlions anglais, tu serais mon cousin, mais si nous parlions espagnol, tu serais mon oncle [En anglais on parle de 'cousin' pour désigner le cousin du père, tandis qu'en espagnol, on parle d'oncle']

(24) SI les transports publics sont la vie d'une ville, Vancouver est dans le coma.

(Exemples adaptés de Dancygier \& Sweetser 2005)

\section{Conditionnels d'actes de discours}

Enfin, nous considérons comme énonciatifs les usages dits d'actes de discours, comme le célèbre exemple d'Austin (exemple25)

(25) SI tu as soif, il y a de la bière dans le frigo

Dans le cas des actes de discours, il s'agit de mettre en place un cadre de pertinence, au sein duquel doit se comprendre la protase : il énonce en quelque sorte une condition du type: si la situation énoncée par la protase est vérifiée, alors voici une information pertinente, autrement, tant pis. Ici, l'apodose est considérée comme vraie indépendamment de la vérité de la protase.

\subsubsection{Cadre hypothétique (valeurs hypothétiques)}

Nous séparons ces valeurs énonciatives, des valeurs hypothétiques qui mettent en jeu des cas où la protase déclenche l'apodose. Cependant, un tel déclenchement peut être factuel ou inférentiel. Dans le premier cas, nous parlerons d'usages hypothétiques prospectifs, et dans le deuxième, d'usages inférentiels.

\section{Les conditionnels hypothétiques prospectifs}

(26) S'il fait beau nous irons nous promener

(27) SI je gagnais au loto, j'achèterais une voiture

Nous regroupons dans cette catégorie les conditionnels prospectifs qui mettent en jeu une notion de prédiction, en présentant la protase comme ayant valeur de potentiel, de possibilité.

\section{Les valeurs inférentielles}

67 Nous souhaitons regrouper au sein des conditionnels hypothétiques des usages qui n'ont pas forcément une valeur prospective, mais dans lesquels la protase déclenche cependant l'apodose, quand bien même ce déclenchement n'est pas un déclenchement factuel mais inférentiel.

Bous regroupons donc les conditionnels hypothético-déductifs (exemples 28 et 29), qui formulent une prédiction qui n'est pas d'ordre temporel ni prospectif, mais déductif, mais aussi les usages souvent considérés comme explicatifs ${ }^{13}$ ou épistémiques ${ }^{14}$ (exemples 30 et $31)$.

(28) SI ABC est un triangle rectangle, alors le carré de son hypoténuse est égal à la somme des carrés de ses deux autres côtés

(29) Ecoute, Antoine, tu diras tout ce que tu veux, moi je sors pas de là : SI tu obliques, tu vas pas tout droit ! (Alain Robbe-Grillet, Les Gommes, 1952) 
(30) si $\mathrm{ABC}$ on triangle à le carré de son hypoténuse égal à la somme des carré de ses deux autres côtés, c'est que c'est un triangle rectangle

(31) S'il lui apporte des croissants le matin, c'est qu'il l'aime ${ }^{15}$ usages épistémiques de Dancygier et Sweetser de mettre en jeu un raisonnement inversé. Cependant, cette notion d'inversion n'a de sens que par rapport à un sens canonique qui nous semble poser problème, particulièrement dans les cas - nombreux - où l'on semble avoir affaire à un raisonnement analytique (au sens kantien). Pour cette raison, nous ne souhaitons pas traiter les usages explicatifs séparément, mais les intégrer à ces valeurs inférentielles.

\section{Remarque sur les usages itératifs}

Dans les usages hypothétiques, on rencontre un grand nombre de cas dans lesquels une itération est énoncée. L'hypothèse porte sur le fait que l'on ne sait pas si dans le cas présent , la protase est réalisée, mais on postule qu'à chaque fois qu'elle l'est, l'apodose l'est aussi. Plus que de prédiction, il est ici question d'une valeur statistique, en tant qu'elle s'appuie sur des précédents avérés, comme dans :

(32) SI je ralentissais, il ralentissait lui aussi.

(33) S'il y a de la lumière dans sa chambre, (c'est que) il est déjà rentré

71 On remarque cependant que ces usages n'excluent ni une valeur prospective (exemple 32), ni une valeur inférentielle (exemple 33). Pour cette raison, on ne considérera pas les usages itératifs comme constituant une classe à part au sein des valeurs hypothétiques, mais juste le caractère hypothétique comme une éventuelle propriété des énoncés hypothétiques en SI.

\subsubsection{Cadre concessif}

72 La troisième classe de phrases en SI que nous voudrions distinguer est celle des phrases concessives. Dans de telles phrases, ce qui est énoncé dans la protase rend possible ce qui est énoncé dans l'apodose malgré la présence d'un enchainement implicite (déjoué) qui affirmerait plutôt SI P, non Q. Il s'agit de poser dans la protase un cadre dans lequel, de façon inattendue en raison des implicatures liées à la protase, l'apodose s'insère, comme dans l'exemple que nous avons cité précédemment.

(1) SI Jake et Chuckie sont les personnages centraux du roman, ce serait faire injure à Mac Liam Wilson d'oublier la palette d'individus tous plus extravagants les uns que les autres. (Critique du roman Eurêka Street de Mc Liam Wilson sur un site Internet consacré à l'auteur)

73 Ainsi, les usages concessifs de SI mettent en jeu une interprétation scalaire (cf. Fauconnier 1975a et b, 1976 et 2003, et Ducrot 1980), qui indique que la condition exprimée par la protase est au-delà des conditions acceptables a priori envisagées pour l'apodose. Elles s'appuient sur un enchaînement de référence qui pose que dans le cas normal, SI P, non $Q$, et énonce le contraire, c'est-à-dire (même) SI P, $Q$.

74 En cela les valeurs concessives du SI mettent en jeu des valeurs qui sont propres à toutes les constructions concessives en bien que, quoique, mais, etc. (cf. Culioli 1999, FilippiDeswelle 1998 et 1999, et Martin 1987).

75 En effet, la relation de concession met en jeu la notion d'une cause inefficace. Ainsi, selon O. Soutet [1990], ce qui est remis en cause dans la concession, ce n'est pas la vérité du 
conséquent, mais sa force argumentative : «le contenu propositionnel est détourné de sa fonction usuelle, à savoir marquer la cohésion discursive, et sert en réalité de repoussoir pour mettre en avant la force argumentative du posé, à savoir $p$ ».

Cette force argumentative repose sur une relation implicite qui lie $P$ à non $Q$, qui constitue un arrière plan commun pour les locuteurs (cf. Morel 1996). Cette relation implicite sur laquelle s'appuie la concession correspond à ce que les culioliens appellent un préconstruit notionnel: il s'agit d'asserter en même temps comme vraies deux propositions qui s'excluent mutuellement en termes de préconstruction notionnelle, tout en indiquant que le locuteur est conscient de la contradiction. La relation de concession fait coexister deux éléments jugés incompatibles, sur la base d'un enchaînement (prédictif ou inférentiel) implicite, que les locuteurs doivent partager pour que la concession soit comprise, en tout cas dans les cas où cette valeur de concession n'est pas marquée linguistiquement, c'est-à-dire dans les constructions en SI qui ne comportent ni même en tête de a protase, ni un marqueur tel que cependant ou malgré tout dans l'apodose.

On peut dès lors envisager les phrases en SI qui ont une valeur concessive comme énonçant une double restriction :

- Une restriction de l'inférence de référence, qui est mise en défaut par la phrase à valeur concessive exprimée

- Une restriction de la portée de la protase, dont l'apodose montre qu'elle ne suffit pas à la prendre en compte. D'une certaine manière, les concessives nous disent que $Q$ échappe à $P$, que la réalisation de l'apodose, contrairement à ce qui était supposé, ne dépend pas de celle de la protase, et ainsi, qu'elles appartiennent en quelque sorte à deux domaines distincts. En un autre sens, elles servent aussi à affirmer avec plus de force l'apodose, qui demeure vraie malgré la protase.

\subsubsection{Cadre comparatif}

Cette classe englobe les propositions contrastives (ou adversatives, pour reprendre les termes de De Vogüe 1986), ainsi que des phrases qui, si elles émettent une comparaison, ne semblent pas pour autant mettre en jeu un contraste, comme :

(34) Je ne nie point que SI vingt métiers font la misère, vingt aventures font la solitude. (Hervé Bazin, La Mort du petit cheval)

Ces phrases comparatives semblent mettre en relation deux propositions sans les placer dans un rapport inférentiel. Ainsi elles se différencient des concessives en tant qu'il n'y est pas vraiment fait référence à une inférence qui serait déjouée. Il s'agit plutôt de créer un parallèle entre la protase et l'apodose, de les placer sur une échelle commune. Le contraste est alors obtenu sémantiquement, par une opposition entre les termes utilisés dans la protase et l'apodose.

Nous voudrions associer ces cas contrastifs aux cas où le SI met en place une comparaison non adversative, comme dans les propositions suivantes où l'échelle est a priori la même ; il s'agit en quelque sorte d'une échelle de sympathie. (cf. schémas 1 et 2)

(35) SI Julie est insupportable, Charles est charmant

(36) SI Julie est insupportable, Charles est pire encore

81 Cependant, l'échelle de départ n'est pas forcément la même, et c'est justement le propre des comparatives que de construire - par le biais de ce que Fauconnier appelle un mapping (cf. Fauconnier 1997) -une échelle commune pour la protase et l'apodose. C'est ce qui se passe dans l'exemple (37). Il s'agit ici de mettre en relation le rapport entre le nombre de 
métiers et la misère, et le nombre d'aventures et la solitude. Une analogie est ainsi posée entre ces deux rapports. L'échelle commune est ici celle du rapport entre un nombre X d'activités (vingt métiers, ou vingt aventures), et l'état (négatif) qui en résulte (en l'occurrence, la misère ou la solitude) ${ }^{16}$.

Il ne s'agit donc pas ici de placer la protase sur une échelle séquentielle ou inférentielle, mais sur une échelle qualitative.

Schéma 1 : SI Julie est insupportable, Charles est charmant

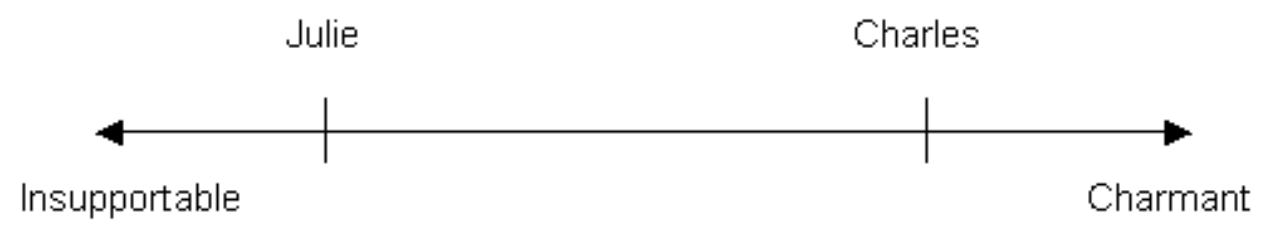

\section{Echelle de sympathie}

Schéma 2 : SI Julie est insupportable, Charles est pire encore

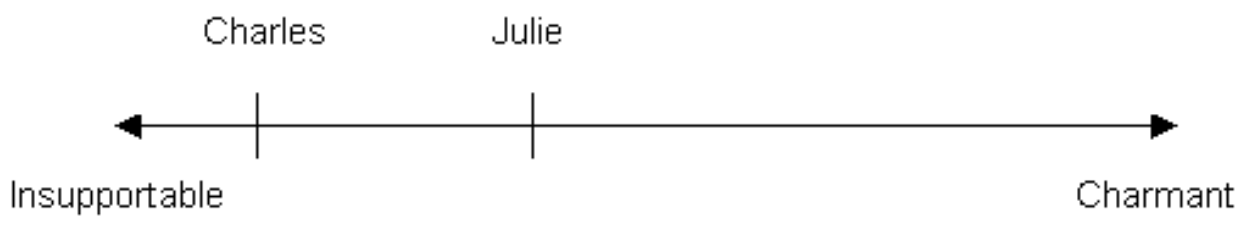

\section{Echelle de sympathie}

83 Nous réunissons donc ici des valeurs qui mettent en jeu un contraste mais aussi des valeurs qui mettent en jeu une comparaison non adversative. En ce qui concerne les valeurs adversatives, elles sont souvent confondues avec les valeurs concessives que nous venons de présenter. Ainsi Muller les présente conjointement au sein des emplois factuels (Muller 1996, p. 214). Pourtant, il nous semble que la différence, liée à la présence, dans le concessives d'un enchaînement implicite déjoué, qui fait défaut dans les adversatives, nous semble les différencier de façon nette. Une telle différenciation est également encouragée par le fait que ces usages n'acceptent pas les mêmes paraphrases, reformulations ou ajouts. Comparons ainsi les deux énoncés suivants :

(37) Même SI Julie est insupportable, Charles est charmant

(37b) SI Julie est insupportable, par contre Charles est charmant

(37) pousse à une interprétation concessive: il semble que le fait que Julie soit insupportable soit implicitement considéré comme devant a priori exclure le fait que Charles soit charmant - en quelque sorte, il découlerait des implicatures de Julie est insupportable le fait que Charles le soit aussi. En revanche, $(24 \mathrm{~b})$ ne semble pas pousser à postuler un quelconque rapport d'enchaînement, qu'il soit supposé ou non, entre le fait que Julie soit insupportable et le fait que Charles soit charmant. Les deux faits sont ici simplement présentés sur une échelle commune. Cette différenciation, qui se fonde sur l'absence dans le cas des comparatives d'une référence à un enchaînement -déjoué ou non - nous semble également justifiée par le fait que la valeur concessive soit compatibles avec des constructions contrefactuelles, ce qui est impossible dans le cas des comparatives : 
(37c) Même SI Julie avait été insupportable, Charles aurait été charmant (concessive)

(37d) SI Julie avait été insupportable, par contre Charles aurait été charmant (hypothétique?)

(37e) SI Julie avait été insupportable, par contre Charles avait été charmant (comparative)

\section{(Fauconnier 1997, 2003) qui permet de mettre en relation $P$ et $Q$. accès à un usage nouveau de $S I$. \\ 2. Locutions associées à SI et marqueurs : compositionnalité et pointeurs.} s'opposent. Mais dans les concessives cette opposition dépend d'un enchaînement supposé qui associe non $Q$ à $P$, tandis que dans les valeurs comparatives, cette opposition est de l'ordre d'une mise en perspective comparative. Par ailleurs, cette idée d'opposition ne concerne au sein des comparatives que les comparatives. Or pour nous les adversatives, comme les comparatives non adversatives, mettent en place une sorte de mapping

Maintenant que nous avons isolé ces valeurs, nous voudrions nous intéresser à la question des marqueurs associés à $S I$, pour déterminer dans quelle mesure ils donnent ou non

Comme nous l'avons noté dans l'étude des valeurs comparatives et concessives, certaines locutions associées à SI, comme même, contraignent l'interprétation : ainsi, la présence de même si nous contraint à comprendre l'énoncé comme mettant en jeu une valeur concessive (exemples 37 et 37c). La présence de même en tête de la protase semble contraindre une interprétation en termes de référence à un enchaînement implicite qui n'est pas à l'œuvre dans les valeurs comparatives. Cependant, de telles valeurs concessives peuvent également être obtenues sans que même figure dans la phrase (exemple 1). Or, certaines constructions en $S I$ ( $Q$, sauf si $P$, ne $Q$ que si $P$ ou encore $P$ seulement si Q) donnent lieu à des usages qui ne pourraient être construit en utilisant uniquement SI, et qui ne sont pas définis au sein des classes que nous venons de présenter. Ce sont ces constructions que nous voudrions présenter, avant de les comparer aux cas où la présence d'un marqueur dans la protase ne fait qu'orienter l'interprétation.

\subsection{Locutions associées à SI qui contraignent l'interprétation}

Nous nous intéresserons donc ici aux cas où la construction d'une locution en SI met en jeu un usage nouveau par rapport aux cas que nous avons présenté, et qui ne peut être 
obtenu dans des phrases dans lesquelles cette locution n'apparaîtrait pas conjointement à SI. C'est le cas de sauf si P, que si ou encore seulement si.

\subsubsection{Constructions en $\mathrm{SI}$ avec sauf : $\mathrm{Q}$, sauf $\mathrm{SI} P$}

On peut construire des propositions exceptives en SI, à l'aide de la locution sauf. Celles-ci marquent l'exclusion d'une possibilité, exprimée par la protase, et manifestent que l'apodose devrait être réalisée, excepté dans le cas où la protase serait réalisée. Elles sont paraphrasables à l'aide de l'expression à moins que + subjonctif, comme le montrent les exemples suivants :

(38) j'irai me baigner, sauf s'il pleut

(386) j'irai me baigner, à moins qu'il ne pleuve

La condition énoncée dans la protase doit être une condition exceptive dans le cadre d'une phrase hypothétique, qui peut être itérative. Ainsi :

(38c) j'allais me baigner, sauf s'il pleuvait

(38d) je vais me baigner, sauf s'il pleut

semblent tout à fait acceptables, alors que des propositions concessives n'acceptent pas la présence de sauf en tête de la protase.

En effet, de telles constructions semblent impliquer la présence d'une condition, et donc d'un enchaînement de la protase à l'apodose. En ceci, elles semblent exclure les usages concessifs, comparatifs et énonciatifs, et mettre en jeu des valeurs hypothétiques.

4 De fait, les usages concessifs semblent devoir poser dans la protase une condition limite (qui pourrait apparemment être exclue) pour l'apodose. Or un tel usage apparait incompatible avec le fait d'exclure une condition, comme le font les phrases en sauf SI.

5 De la même façon, les propositions comparatives ne semblent pas pouvoir accepter la présence de sauf, qui donne à la phrase une valeur conditionnelle absente des énoncés comparatifs.

6 De même, les constructions d'actes de discours en SI, en tant que la protase en sauf SI semble devoir fournir un cadre de réalisation pour l'apodose, alors que les conditionnels d'actes de discours sont au contraire caractérisés par le fait que l'apodose est réalisée, quelle que soit la valeur de la protase. En ce qui concerne les valeurs énonciatives qui ne sont pas des actes de discours, elles partagent avec ces dernières la propriété d'énoncer l'apodose quoiqu'il en soit, malgré les restrictions éventuellement fournie dans la protase.

Cependant, au sein des valeurs que nous avons qualifiées d'hypothétiques, le cas des usages explicatifs semble poser problème. En effet, si que ceux-ci manifestent bien une forme d'enchaînement, cet enchaînement semble aller plutôt de l'apodose vers la protase.

Examinons l'exemple suivant :

(39) ?? sauf SI la lumière est éteinte, c'est qu'ils sont là

Un tel exemple ne semble pas acceptable tout seul. Cependant, si l'on construit un exemple dans lequel la proposition en sauf SI précise les conditions de vérité de l'apodose, exprimée dans une première proposition en $S I$, un tel usage semble possible, comme l'illustre la série d'exemples suivante :

(39b) SI la lumière est allumée, c'est qu'ils sont là, sauf s'ils ont oublié de l'éteindre

(39c) SI la lumière est allumée, c'est qu'ils sont là, s'ils n'ont pas oublié de l'éteindre.

(39d) SI la lumière est allumée, c'est qu'ils sont là, à moins qu'ils aient oublié de l'éteindre. 

en sauf SI.

Il semble que dans de tels cas, la subordonnée en sauf SI ne puisse que compléter une proposition explicative, en excluant un cas qui invaliderait le raisonnement posé par celle-ci. Dès lors, il s'agit bien de restreindre d'excepter une condition, mais à partir d'une condition générale énoncée par la protase. Les usages explicatifs doivent en effet proposer dans l'apodose une raison pour la protase, et il ne peut donc s'agir d'en exclure une avant même qu'une hypothèse ait été formulée.

Ainsi, les constructions en sauf SI semblent d'une manière générale marquer une valeur de rectification de l'apodose. Cela explique qu'elles soient plus souvent utilisées des cas où la subordonnée est placée après la principale, même s'il est possible de trouver la protase en tête de phrase. La valeur à accorder aux expressions en sauf SI semblent déductible de manière compositionnelle à partir des valeurs de sauf et de SI. La grande compositionnalité du sens de ces expressions nous a fait décider de ne pas les inclure dans notre classification ses constructions en SI. L'effet de sens obtenu grâce à sauf SI ne peut en effet se retrouver dans des phrases construites seulement avec SI.

\subsubsection{Les propositions en $\mathrm{SI}$ avec que (ne $\mathrm{Q}$ que $\mathrm{SI} P$ ) et avec seulement ( $\mathrm{P}$ seulement SI Q)}

Les phrases en ne Q que SI P semblent faire pendant à celles en sauf SI, en cela qu'au lieu d'exclure une condition, elles en isolent une qui est considérée comme la seule dans laquelle l'apodose, placée avant la protase, pourrait se réaliser.

Ainsi, on peut paraphraser (38) en (38e)

(38) j'irai me baigner, sauf s'il pleut

(38e) je n'irai me baigner que s'il ne pleut pas.

Cependant, tandis que les propositions en sauf SI semblent introduire une condition suffisante (qui correspond à $\boldsymbol{n} \boldsymbol{e} \mathbf{P}$ ) pour la réalisation de l'apodose, les propositions en que $S I$ introduisent quant à elles une condition nécessaire ${ }^{17}$ (qui correspond cette fois à $P$ ) pour la réalisation de $\boldsymbol{n} \boldsymbol{Q}$, comme le montrent les exemples suivants :

(38d) je vais me baigner, sauf s'il pleut, qui semble impliquer que le locuteur va se baigner à chaque fois qu'il ne pleut pas

(38f) je ne vais me baigner que s'il ne pleut pas, qui ne signifie pas qu'il se baigne dès lors qu'il ne pleut pas, mais qu'il ne se baigne pas dès lors qu'il pleut.

Ainsi, si les phrases en que SI semblent partager avec celles en sauf SI l'impossibilité d'exprimer des valeurs contrastives ou concessives, en revanche, elles ne semblent pas poser les mêmes problèmes concernant la possibilité d'avoir une valeur contrefactuelle. En effet, l'apodose conserve une valeur beaucoup plus hypothétique que dans les énoncés

Elles semblent de plus devoir être mise en relation avec les phrases en seulement SI, qui introduisent elles aussi une condition nécessaire, mais ne se construisent pas avec la négation qui intervient dans l'apodose des phrases en sauf SI, comme le montrent les exemples suivants :

(40) Non, monsieur, répondit Ned Land, car cette bête-là ne se laissera prendre que SI elle le veut bien. (Jules Verne, Vingt mille lieues sous les mers)

(40b) Non, monsieur, répondit Ned Land, car cette bête-là ne se laissera pas prendre , sauf SI elle le veut bien.

(40c) Non, monsieur, répondit Ned Land, car cette bête-là se laissera prendre seulement SI elle le veut bien. 
(41) En général de telles comparaisons ne peuvent être satisfaisantes que SI les conditions géographiques des régions comparées ne sont pas trop différentes, par exemple s'il s'agit de diviser en régions climatiques un pays d'une certaine étendue, comme la France. (Charles Maurain, La Météorologie et ses applications, 1950)

(41b) En général de telles comparaisons ne peuvent pas être satisfaisantes, sauf SI les conditions géographiques des régions comparées ne sont pas trop différentes, par exemple s'il s'agit de diviser en régions climatiques un pays d'une certaine étendue, comme la France.

(41c) En général de telles comparaisons peuvent être satisfaisantes seulement SI les conditions géographiques des régions comparées ne sont pas trop différentes, par exemple s'il s'agit de diviser en régions climatiques un pays d'une certaine étendue, comme la France.

Ainsi, dans les phrases en sauf SI, la négation est généralement complète dans l'apodose il s'agit d'exclure un condition pour la réalisation de la protase. Si la protase $(P)$ n'est pas réalisée (si donc non $P$ est réalisé), alors cela suffit à ce que l'apodose $(Q)$ soit réalisée. La présence de sauf donne une valeur négative à la protase.

Dans les phrases en que SI, en revanche, la protase est directement présentée comme une condition en elle-même (on ne s'intéresse pas à sa négation): il s'agit de poser la condition exprimée par la protase comme une condition nécessaire à la réalisation de l'apodose, qui comporte en général une trace de négation, mais pas une négation complète (ainsi, on trouve généralement seulement le ne, et pas ne... pas). Cette trace de négation n'implique pas que l'apodose soit prise dans un sens négatif. Ainsi, (40) et (41) sont à comprendre respectivement ainsi :

(40d) Le fait qu'elle le veuille bien est une condition nécessaire au fait qu'elle se laisse prendre

(41d) En général, le fait que les conditions géographiques des régions comparées ne soient pas trop différentes est une condition nécessaire pour que de telles comparaisons soient satisfaisantes.

110 La valeur non négative du ne est rendue plus manifeste par le fait que la négation complète en ne...pas n'est pas possible, comme le montre les exemples suivants :

(40e) *Non, monsieur, répondit Ned Land, car cette bête-là ne se laissera pas prendre que SI elle le veut bien.

(41e) *En général de telles comparaisons ne peuvent pas être satisfaisantes que SI les conditions géographiques des régions comparées ne sont pas trop différentes, par exemple s'il s'agit de diviser en régions climatiques un pays d'une certaine étendue, comme la France.

111 Cette valeur non négative est plus flagrante dans les phrases en seulement SI, comme dans les phrases (40c) et (41c).

Si l'on essaye de transcrire ces constructions en sauf SI, que SI et SI seulement dans les termes de la logique classique, on remarque qu'elles permettent d'exprimer différents types de conditionnels classiques. Ainsi, la protase, introduite par SI ne correspond pas forcément à l'antécédent d'une implication matérielle - ce que nous avons déjà relevé en parlant de condition suffisante pour les phrases en sauf SI, et de condition nécessaire dans le cas des phrases en que SI ou seulement SI. En effet, dans l'implication matérielle de la logique classique, l'antécédent de l'implication matérielle correspond à la condition suffisante de son conséquent, qui lui-même est la condition nécessaire de l'antécédent. Ainsi, dans une proposition $p_{\mathbb{E}}^{\mathbb{E}} q, p$ est condition suffisante de $q$, et $q$ est condition nécessaire de $p$. 
Reprenons l'exemple (38), et examinons (de nouveau) ses transformations au présent qui emploient que SI, et seulement SI :

(38d) Je vais me baigner, sauf s'il pleut

(38i) Je ne vais me baigner que s'il ne pleut pas

(38j) Je vais me baigner seulement s'il ne pleut pas

Si on représente il pleut par $P$ et je vais me baigner par $Q$, on peut représenter ainsi les trois propositions précédentes :

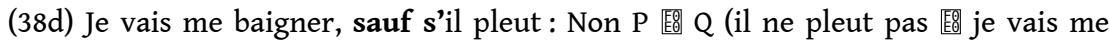
baigner)

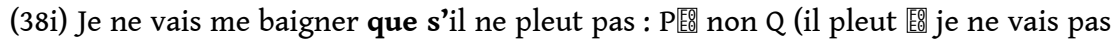
me baigner)

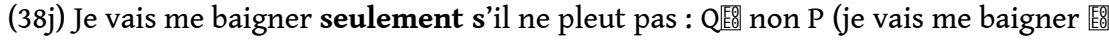
il ne pleut pas)

En effet, la phrase (38d) exprime que non $P$ est une condition suffisante de $Q$ (il ne pleut pas est une condition suffisante de je vais me baigner), ce qui correspond, selon la logique classique, au fait que $Q$ est une condition nécessaire de non $P$ (je vais me baigner est une condition nécessaire de il ne pleut pas). Dans (38i) en revanche, c'est $P$ qui est une condition suffisante de non $Q$, ce qui correspond, selon la logique classique ${ }^{18}$, à dire que $Q$ est une condition suffisante de non $P$ (ce qui est aussi exprimé par (38j)). Ainsi, du point de vue de la logique classique, (38i) et (38j) sont donc équivalentes, et toutes deux correspondent à l'implication inverse de celle exprimée dans (38d).

Ainsi, les phrases en que SI et seulement SI semblent bien devoir être comprises comme faisant pendant à celles en sauf SI, en cela qu'elles expriment dans la protase les cas dans lesquels l'apodose peut éventuellement se réaliser, tandis que les phrases en sauf SI expriment dans la protase les (seuls) cas dans lesquels l'apodose ne peut pas se réaliser.

117 Ainsi, il semble que le comportement de seulement en français soit assez proche de celui de only en anglais. En effet, comme le soulignent Dancygier et Sweetser, only modifie «conventionnellement et explicitement » la «structure inférentielle de la construction de l'espace conditionnel ». Selon ces auteures, only s'accorde compositionnellement avec la sémantique de la phrase selon sa relation de portée avec la proposition. Dans le cas standard de only if, only précède et porte sur toute la proposition en if. Dès lors le sens compositionnel serait que « $Q$ est vrai (holds) 'seulement dans l'espace $P$ ', et pas dans d'autres espaces ", tandis que if only « présente des problèmes d'interprétation scopale et de polysémie de only beaucoup plus complexes » (Dancygier et Sweetser 2005, 209-11).

Selon Dancygier \& Sweetser, dans les cas où if précède only, « on ne nous dit pas qu'il n'y a qu'une seule caractéristique pertinente de l'espace en question, mais que parmi les espaces, seul cet espace est un espace acceptable ». Cependant, il ne s'agit pas non plus de dire qu'il n'existe qu'une seule option possible, mais il faut plutôt comprendre only comme « assertant la négation des options rejetées, et présupposant la vérité de l'option sélectionnée » (cf. Dancygier et Sweetser 2005 qui suivent sur ce point Horn 1969). Un conditionnel en only if met en avant l'exclusivité de l'espace défini par $P$ comme une mise en place pour $Q$. les constructions en only if construisent un nouvel espace qui sert d'arrière plan à un espace $Q$ déjà construit.

D'une manière générale, les phrases en seulement SI mettent en jeu une construction scalaire ${ }^{19}$. Examinons l'exemple suivant : 
(42) Ils seront en mesure d'attirer de nouvelles infirmières seulement SI le gouvernement les paye 40 euros de l'heure. (Adapté de Dancygier et Sweetser 2005) 20 Nous voudrions désormais faire un point sur la différence entre ces phrases en seulement SI et les constructions en SI seulement.

Celles-ci correspondent aux usages qui apparaissent dans les exemples suivants :

(43) Je reconnais la voix lente de Boulier : «SI seulement c'était vrai, qu'on soye tranquille ». (M. Genevoix, Ceux de 14)

(44) - SI seulement j'en avais vu! (M. Genevoix, Ceux de 14)

(45) Je l'ai bien senti, allez, derrière l'arbre qui m'cachait : SI seulement j'avais montré ma tête, c'était moi qu'étais déglingué. (M. Genevoix, Ceux de 14)

s'agit ici non plus de poser une condition nécessaire, mais d'indiquer par la présence de seulement après SI qu'il s'agit d'un souhait, exprimé par la protase (exemples (43) et (44)) ou d'une condition limite, en tant qu'elle apparait à la fois minimale et suffisante. C'est le cas de l'exemple (45), qui pourrait être paraphrasé par les deux phrases suivantes :

(45b) Je l'ai bien senti, allez, derrière l'arbre qui m'cachait : il aurait suffi que je montre ma tête, et c'était moi qu'étais déglingué.

(45c) Je l'ai bien senti, allez, derrière l'arbre qui m'cachait : à peine aurais-je montré ma tête, c'était moi qu'étais déglingué.

Dans les cas où le seulement SI exprime un souhait, la présence de l'apodose n'est pas nécessaire, et elle est le plus souvent implicite. Ces constructions sont souvent à l'imparfait, et la valeur de souhait est ainsi doublée d'une valeur de distance, qui indique que ce souhait correspond à un état de fait qui apparaît comme difficilement atteignable, ou loin de la réalité présente. Ainsi, c'est non seulement la locution seulement SI qui 
permet une compréhension à valeur de souhait, mais cette construction temporelle distanciée. Contrairement à ce que nous avions pour seulement SI, dans les phrases en seulement SI, la protase précède volontiers l'apodose, qui peut être absente.

Ce que nous voudrions mettre en avant, c'est la différence entre SI seulement et seulement SI. Ces deux locutions ont des comportements différents tant du point de vue de leurs portées que de celui de leur degré de compositionnalité. Ainsi, si l'on s'en tient à la notion de compositionnalité, on s'attend à ce que SI seulement se comprenne comme construisant un espace $P$, grâce au SI, dans lequel seul X est vrai, en tant que le SI précède le seulement, et qu'il est donc supposé l'englober dans sa portée. Cependant, la forte corrélation de ces constructions avec des phrases qui expriment des souhaits semble remettre en cause cette approche compositionnelle. Cela nous semble pouvoir s'expliquer par la nécessité dans les cas de l'expression d'un souhait d'utiliser de formes distanciées.

Ainsi, il semble que cette valeur de souhait ne puisse se dégager que d'une construction distanciée, soit temporellement dans le futur, soit épistémiquement grâce à l'usage de l'imparfait ou du plus que parfait.

Cependant, les valeurs de souhait ne sont pas les seules à être possibles pour les usages de SI seulement, comme le montre l'exemple (45). Dans cet exemple, il semble que la compositionnalité que nous avons dégagée se maintient : il $\mathrm{y}$ est dit que dans le cas où l'espace de la protase est construit, alors, seule l'évènement exprimé par l'apodose peut être vrai. L'usage de l'imparfait ne correspond pas ici à une forme distanciée, mais seulement à un récit au passé. Pour autant, le fait que de telles valeurs de souhaits soient possibles, nous invite à remettre en cause le simple principe de compositionnalité, ou du moins à considérer que celui-ci ne fonctionne pas comme une simple compositionnalité lexicale, mais que s'y ajoute la polysémie temporelle que nous avons déjà évoquée.

\subsection{Marqueurs dans la protase qui orientent l'interprétation}

131 La comparaison entre les propositions en SI seulement et les propositions en seulement SI nous donnent à penser qu'il y a une différence essentielle entre ces deux cas : dans le premier cas, une valeur exceptive est construite, qui ne saurait être construite sans le recours à une locution en plus de SI, tandis que dans le deuxième cas, seulement permet plutôt de contraindre l'interprétation hypothétique. Après avoir présenté des cas de locutions, comme si seulement, nous voudrions maintenant nous intéresser à ce deuxième cas, où les propositions en SI comportent des marqueurs, ou des pointeurs, qui orientent l'interprétation. C'est le cas par exemple de jamais et de même.

\subsubsection{Les propositions en $\mathrm{SI}$ avec jamais : $\mathrm{SI}$ jamais $\mathrm{P}, \mathrm{Q}$}

On trouve un grand nombre de propositions en SI construites avec jamais. Nous voudrions montrer que la présence de jamais marque une valeur de potentialité associée à la protase, c'est-à-dire une possibilité qui pourrait ou aurait pu être vérifiée. La présence de jamais pose un doute sur la possibilité de la réalisation de la protase, qui a valeur d'irréel (au passé) ou de potentiel distancié (au présent).

Ainsi, la proposition (46) se différencie de la même phrase sans jamais en tant qu'elle semble poser comme improbable la réalisation de la protase, ce qui est beaucoup moins appuyé dans : 
(46) SI jamais cela lui arrive de s'éprendre d'une femme, il sera très ennuyeux. (J.

Green, Moïra)

(46b) SI cela lui arrive de s'éprendre d'une femme, il sera très ennuyeux. de conditions implicites, et indiquent que la condition exprimée par la protase est la dernière à permettre l'apodose, ou plus exactement qu'elle est au-delà des conditions acceptables a priori envisagées. En termes d'espaces mentaux, cela correspond au fait que l'espace de la protase est considéré par rapport à un ensemble d'espaces qui sont considérés comme permettant l'apodose, et que cet espace de $P$ doit être considéré comme au-delà de ces espaces. Ainsi, les phrases en même SI posent une condition qui 
semble a priori ne pas permettre l'apodose, et qui la permet cependant. Cette condition se trouve ainsi englober toutes les précédentes, en tant qu'elle est considérée comme plus forte.

Examinons ainsi l'exemple suivant :

(51) même s'il commet un crime, ils voteront pour X [Cet exemple est repris de l'analyse de Dancygier et Sweetser [2005], s'appuie plus généralement sur les études de G. Fauconnier sur les constructions scalaires (Fauconnier 1975a et b, 1976 et 2003)]

Cette phrase doit être comprise comme signifiant non seulement que les gens voteront pour X s'il commet un crime, mais par là même que si X ne commet pas de crime, ils voteront pour lui.

Ainsi, tandis que les phrases en seulement SI que nous avons examinées mettaient en scène une condition minimale dans laquelle l'apodose serait réalisée, les phrases en même SI mettent en jeu une condition maximale dans laquelle l'apodose serait réalisée. Il s'agit donc ici d'englober dans l'ensemble des espaces permettant l'apodose un espace qui en était a priori exclu.

Cette construction scalaire met ainsi en jeu une chaine causale implicite de non $P$ à $Q$. en effet, ce qui est présupposé dans un tel exemple, c'est la présence d'une inférence «normale » à laquelle la phrase se réfère, et qui consiste à penser que, normalement, si $\mathrm{X}$ commet un crime, les gens ne voteront pas pour lui. Les constructions en même SI posent dans le même temps cette enchaînement de référence, et construit une relation anormale, inattendue, dans laquelle cet enchaînement est déjoué.

147 Ainsi, dans :

(52) - Puisque je te le jure, même SI je voulais te laisser, je ne pourrais pas. ( Marguerite Duras, Un barrage contre le Pacifique)

148 l'inférence implicite est que le locuteur ne pourrait pas laisser le destinataire de la phrase s'il ne le voulait pas, mais que même dans le cas où il le voudrait, il en serait incapable. Il en va de même dans les exemples suivants :

(53) Même SI ta mère n'avait pas envoyé le mot je serais passé.

(54) Même SI je descends jusqu'en enfer, le bras de Dieu est assez long pour m'en retirer. (J. Green, Journal)

La présence de l'inférence implicite, qui tient du bon sens, explique aussi pourquoi on peut construire des phrases en SI concessives sans la présence de même. En effet, la valeur concessive vient alors d'une contradiction entre le conditionnel exprimée et une inférence causale supposée connue des destinataires du discours. Ainsi, l'exemple (1) que nous avions donné se comprend de manière concessive bien que même n'y figure pas :

(1) SI Jake et Chuckie sont les personnages centraux du roman, ce serait faire injure à Mac Liam Wilson d'oublier la palette d'individus tous plus extravagants les uns que les autres. (Critique du roman Eurêka Street de Mc Liam Wilson sur un site Internet consacré à l'auteur)

150 En effet, l'inférence implicite selon laquelle le fait que Jake et Chuckie soient les personnages centraux du livre pourrait faire oublier les autres personnages est suffisamment présente pour qu'on comprenne que la protase se pose comme une condition maximale et inattendue.

151 Les constructions en même SI se distinguent des constructions en seulement SI, sauf SI ou que SI. En effet, l'effet de sens obtenu par l'association de même à SI semble pouvoir être obtenu sans la présence de même, qui ne fait que l'accentuer, ou rendre obligatoire une 
interprétation. En revanche, les valeurs obtenues dans des constructions en seulement SI, sauf SI ou que SI semblent impossibles dans des phrases où seul le SI serait présent. En ce sens, ces locutions apparaissent compositionnelles : l'instruction restrictive charriée par que, seulement, et (de manière différente) par sauf inclut dans sa portée le SI qui ne s'applique que sous cette restriction. Le cas de même SI et de SI jamais est un peu différent.

Le cas de même SI se rapproche de celui de SI jamais. En effet, SI jamais permet également de contraindre une interprétation, mais les valeurs que cette locution génère semble pouvoir être exprimées y compris en l'absence de jamais. Il met en place un espace présenté comme lointain par rapport au monde actuel. Il exprime ainsi une sorte de valeur modale, qui pourrait être paraphrasée par une expression du type « mais c'est peu probable ».

\section{Conclusion}

Ainsi, les constructions en seulement SI, sauf SI ou que SI semblent mettre en place des valeurs du SI qui ne sont pas envisageables en leur absence, tandis que SI jamais et même SI semblent plutôt contraindre une interprétation. On les considérera donc comme des marqueurs renvoyant à des valeurs déterminées de SI, tandis que les constructions en seulement SI, sauf SI ou que SI seront considérées comme des locutions différentes, dont l'interprétation doit se comprendre comme un tout, résultant compositionnellement de l'association de si et du terme qui lui est adjoint. Cependant, le fait que seulement si et si seulement ne s'interprètent pas de la même façon nous invite à penser que la question de la portée de SI rentre en jeu, et que si seulement ne met pas en jeu une réelle compositionnalité, mais permet plutôt de pointer vers l'une des classes d'usages que nous avons définie, ou plutôt d'exclure les autres classes, en présentant l'apodose comme une hypothèse non réalisée. Ainsi, cette différenciation entre des cas de compositionnalité, qui permettent la formation de locutions (donnant lieu à des usages nouveaux) et des cas de renforcement d'une valeur de SI grâce à des marqueurs nous semblent fournir une validation de notre classification en manifestant que les usages pointés par des marqueurs correspondent à des cas pris en compte par celle-ci, tandis que les cas de locutions correspondent à des usages nouveaux.

\section{BIBLIOGRAPHIE}

Charolles, M. (2003), « De la topicalité des adverbiaux détachés en tête de phrase », in CHAROLLES, M. Et PREVOST, S. (éds.) (2003), Adverbiaux et topiques, Louvain la Neuve, Travaux de Linguistique, 47, pp. 11-51.

Culioli, A. (1999), Pour une linguistique de l'énonciation. Formalisation et opérations de repérage, tome 2, Paris, Ophrys.

Culioli, A. , (1999), "Even though, even if; as though, as if", in Pour une linguistique de l'énonciation, tome 1, Paris, Ophrys. 
Dancygier, B. (1988) Conditionals and concessives, Papers and Studies in Cognitive Linguistics 24, p. $123-32$

Dancygier, B. (1993), “Interpreting Conditionals: Time, Knowledge and Causation, Journal of Pragmatics 19, p. 403-34

Dancygier, B. \& Sweetser, E. (2005), « Mental spaces in Grammar. Conditional constructions”, Cambridge Studies in Linguistics, n.108, Cambridge, Cambridge University Press.

Degand, L. \& Pander Maat, H. (2003), “A contrastive study of Dutch and French causal connectives on the Speaker Involvement Scale”, in VERHAGEN, A., \& VAN DE WEIJER, J. , (éd.), Levels in Language and Cognition: Lexicon, Grammar, Discourse.

Degand, L. \& Pander Maat, H. (1999), "Scaling causal relations in terms of Speaker Involvement, Levels of Representation in Discourse", Working Notes of the International Workshop on Text Representation, Edinburgh University, pp. 45-54.

De Vogüé, S., (1986-1987), "La conjonction “si" et la question de l'homonymie", in BULAG, n.13, Université de Besançon.

De Vogüé, S., (1990), "Inférence sous inférence", Le gré des langues, n.1, octobre 1990, Paris, L'harmattan, pp. 7-24.

De Vogüé, S., (1992), "Aux frontières des domaines notionnels: "bien que", “quoique" et "encore que"", L'information grammaticale, 55, octobre 1992, Paris, Baillière, pp.23-27.

De Vogüé, S. (1999), « Le Champ des subordonnées dites conditionnelles du français : conditions, éventualités, suppositions et hypothèses ", in TREVISE, A., L'hypothétique, LINX, n. 41, Paris X Nanterre, pp. 93-118.

De Vogüé, S., (2004), «Si, au centre et aux marges de la condition », in Hare, C., L'hypothèse au miroir des langues, Paris, L'Harmattan, p. 85-117.

Ducrot, O. (1971), "L'expression, en français, de la notion de condition suffisante", in Langue Française, n.12, pp. 60-67.

Ducrot, O. (1980), Les échelles argumentatives, Paris, Minuit.

Fauconnier, G. (1975), "Pragmatic scales and logical structure”, in Linguistic Inquiry, n. 6, pp. 353-375.

Fauconnier, G. (1976), « Remarques sur la théorie des phénomènes scalaires », in Semantikos, I-3, pp. 13-36.

Fauconnier, G. (1985), Mental spaces: Aspects of meaning construction in natural language, Cambridge, Cambridge University Press.

Fauconnier, G.(1997), Mappings in Thought and Language, New York, Cambridge University Press.

Fauconnier, G. (2003), "Polarity and the Scale Principle" in GUTIERREZ-REXACH, J. (éd.), Semantics: Critical Concepts in Linguistics, Londres et New York, Routledge.

Fauconnier, G., Turner M. (1996), « Blending as a central Process of Grammar» in A. Goldberg, (éd), Conceptual Structure, Discourse, and Language, Stanford, CLSI Publications.

Fauconnier, G. et Turner M. 2002, The Way We Think. Conceptual Blending and the Mind's hidden Complexities. New York, Basic Books 
Filippi, C., (1998), "La relation de concession : étude contrastive de though en anglais et de bien que, même si et encore que en français", Linguistique contrastive et traduction, n.4, Paris, Ophrys.

Fillmore, C. J. (1990a), "Epistemic stance and grammatical form in English conditional sentences", in CLS, n. 26, pp. 137-162.

Fillmore, C. J. (1990b), “The contribution of linguistics to langage understanding”, in BOCAZ A. (éd.), Proceedings of the First Symposium on Cognition, Langage and Culture, Universidad de Chile, pp.109-128.

Hare, C. (2005), L'hypothèse au miroir des langues, Paris, L'Harmattan.

Iatridou, S. (1991), Topics in Conditionals, PhD dissertation, MIT, Cambridge, Massachusetts. Distributed by MIT Working Papers in Linguistics

Iatridou, S. (1994) "On the Meaning of Conditional then" in Natural Language Semantics 2, pp.171-199.

Iten, C., (2002). "Even if and Even: The Case for an Inferential Scalar Account”, UCL Working Papers in Linguistics 14, 120-157

Martin R. (1987), Martin, langage et croyance, Mardaga, Bruxelles

Muller, C. (1993), « Si comparatif, consécutif, concessif exclamatif », n G. HILTY et alii (éd), Actes du XXe congrès International de Linguistique et Philologie Romane, Tome 1, FranckeTübingenBâle, 551-562

Muller, C., (1994a), « Combien faut-il distinguer de si » in A. IBRAHIM (éd) Supports, opérateurs, durées, Annales de Lettres de Besançon, 516, Diffusion Les Belles Lettres, Paris 185-206

Muller, C., (1996), La subordination en français, Paris, Armand Colin

Piot, M., (2002), « La conjonction "même si" n'existe pas! », in Laporte, E.,

Leclere, C., Piot, M. \& Silberztein, M. (éd.), Syntaxe, Lexique

et Lexique-grammaire, Volume dédié à Maurice Gross, Linguisticae Investigationes

Supplementa , n. 24, Amsterdam et Philadelphie, John Benjamins Publishing \& Co.

Soutet, O., (1990) La concession en français des origines au XVIème siècle. Problèmes généraux . Les tours prépositionnels, Genève, Droz

Sweetser, E. (1996a), Reasoning, Mappings and Meta-metaphorical conditionals in Shibatani M. \& Thompson, S. (éd) Grammatical constructions : their form and meaning, Oxford, Oxford University Press.

Victorri, B., \& Fuchs, C., (1996), La polysémie - Construction dynamique du sens, Hermès.

Victorri, B., (1999), « Le sens grammatical », in Langages, n.136, vol. décembre, n. spécial

"Sémantique lexicale et grammaticale", pp. 85-122.

\section{ANNEXES}

\section{Romans :}

- L'abbé C, (Georges Bataille) 
- La Mort du petit cheval (Hervé Bazin)

- Un barrage contre le Pacifique (Marguerite Duras)

- Armance (Stendhal)

- Vingt mille lieues sous les mers (Jules Verne)

- Allons z'enfants (Yves Gibeau)

- Les Gommes (Alain Robbe-Grillet)

- Touchez pas au grisbi (Albert Simonin)

- L'Arrache-cœur (Boris Vian)

\section{Rapports :}

- L'enseignement en France : l'enseignement de la musique et l'éducation musicale. (1950)

- L'industrie des conserves en France (1950)

- Les Musées en France (1950)

\section{Essais :}

- Les Sentiers et les routes de la poésie (Paul Eluard)

- Le Degré zéro de l'écriture (Roland Barthes)

- Problèmes de la danse (Maurice Brillant)

- Études de psychologie médicale (Jean Delay)

\section{NOTES}

1. Piot 2002

2. Piot 2002.

3. Morel 1996, Ericksson 1985, Sundell 1985

4. cf. Victorri et Fuchs, 1996.

5. Nous reviendrons bien évidemment sur toutes ces valeurs, dont nous donnerons une présentation détaillée dans la suite du texte.

6. Voir détails en bibliographie

7. http://atilf.atilf.fr/frantext.htm

8. Cette idée de prise en charge de la protase correspond à la notion d'Epistemic stance développée par Fillmore (Fillmore 1990) et utilisée par Sweetser et Dancygier. Elle reprend également les théories développées par Pander Maat et Degand sur les constructions en puisque, qui font référence à l'idée d'une échelle (continue) de prise en charge par le locuteur, mais cette fois-ci il s'agit de la prise en charge d'une relation, et non plus d'une proposition, comme dans le cas présent. Pander Maat et Degand 1999 et 2000.

9. Fillmore, 1990a

10. Dancygier et Sweetser 2005, p 43 sq.

11. On peut rapprocher cette notion de la notion d'enchaînement développée par A. Culioli qui l'utilise au sujet des conditionnels. Culioli 1999. 
12. Nous aurons l'occasion de revenir sur ces catégories qui seront reprises dans notre classification

13. Cf. De Vogüe 1986.

14. Cf. Dancygier et Sweetser 2005.

15. Cet exemple est adapté de Dancygier et Sweetser 2005.

16. De tels cas où l'échelle ne correspond finalement ni à celle de la protase ni à celle de l'apodose sont plus facilement abordables en termes de blendings que de simples mappings. Fauconnier et Turner 1996, 2002.

17. Pour une étude de la relation des constructions en si à la notion de condition nécessaire et à celle de condition suffisante, voir De Vogüe 2001.

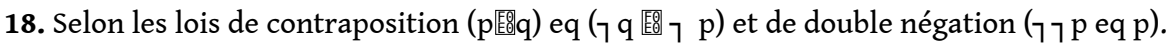

19. G. Fauconnier, 1975.

20. On remarque que cet exemple est équivalent à l'exemple suivant :

"Ils ne seront en mesure d'attirer de nouvelles infirmières que si le gouvernement les paye $38 \$$ de l'heure ", où l'on utilise une construction en "que si ", et que l'analyse en termes scalaires que nous développons pour les phrases en «seulement si » peut être appliquée aux constructions en "que si ».

21. Cette notion d'échelle est à rapprocher de celle mise en place dans les usages concessifs, à ceci près qu'ici on prend l'extrémité inverse de l'échelle.

\section{RÉSUMÉS}

Nous nous intéresserons ici à des cas dans lesquels SI est associé à un autre marqueur, pour essayer de déterminer les différents types d'usages qui s'en dégagent. En nous appuyant sur notre classification des valeurs de SI, nous distinguerons deux cas. Dans le premier cas ( $Q$, sauf si $P$, ne $Q$ que si $P$ ou encore $P$ seulement si $Q$ ), il semble qu'un sens nouveau émerge, qui ne pourrait être construit uniquement avec SI, et qui semble même pouvoir aller à l'encontre des usages traditionnels de SI (comme par exemple pour sauf SI). Dans le deuxième cas au contraire $(Q$, si seulement $P$ ou si seulement $P, Q$, même si $P, Q$, ou si jamais), la présence du deuxième marqueur ne semble pas faire émerger un sens nouveau par rapport aux usages que nous avons pu dégager pour SI, mais plutôt pointer vers un usage déterminé du SI qui pourrait être construit en leur absence, ou l'accentuer.

In this paper, we would like to focus on cases in which the French conditional marker SI is associated with some other markers, in order to determine what kind of uses are induced by these constructions. According to our classification of SI uses, we will distinguish two cases. In the first case $(\mathrm{Q}$, sauf si $\mathrm{P}$, ne $\mathrm{Q}$ que si $\mathrm{P}$ and $\mathrm{P}$ seulement si $\mathrm{Q})$, we consider that a new use emerges that could not be built using only SI. On the contrary, in the second case $(Q$, si seulement $P$, si seulement $P, Q$, même si $P, Q$, and si jamais), the second marker does not lead to a new use of SI. On the contrary, it seems to work as an indication to decide which use of SI the speaker refers to by pointing to an existing use of SI that could be built without using this marker. 
INDEX

Keywords : conditional, semantics, cognitive grammar, compositionality, instructional meaning Mots-clés : conditionnel, sémantique, grammaire cognitive, compositionnalité, sens instructionnel

\section{AUTEUR}

\section{JEANNE APTEKMAN}

Université Blaise Pascal, Clermont-Ferrand 2, Lattice UMR 8094 (ENS-CNRS) 